\title{
BMJ Open Effect of deinstitutionalisation on quality of life for adults with intellectual disabilities: a systematic review
}

\author{
Mary McCarron, ${ }^{1,2}$ Richard Lombard-Vance, ${ }^{3}$ Esther Murphy, ${ }^{3}$ Peter May, ${ }^{\oplus 4,5}$ \\ Naoise Webb, ${ }^{6}$ Greg Sheaf, ${ }^{7}$ Philip McCallion, ${ }^{2,8}$ Roger Stancliffe, ${ }^{9}$ \\ Charles Normand, ${ }^{4,10}$ Valerie Smith, ${ }^{3}$ Mary-Ann O'Donovan ${ }^{11}$
}

To cite: McCarron M, LombardVance R, Murphy E, et al. Effect of deinstitutionalisation on quality of life for adults with intellectual disabilities: a systematic review. BMJ Open 2019:9:e025735. doi:10.1136/ bmjopen-2018-025735

- Prepublication history and additional material for this paper are available online. To view these files, please visit the journal online (http://dx.doi. org/10.1136/bmjopen-2018025735).

Received 2 August 2018 Revised 22 November 2018 Accepted 15 January 2019

Check for updates

(C) Author(s) (or their employer(s)) 2019. Re-use permitted under CC BY-NC. No commercial re-use. See rights and permissions. Published by BMJ.

For numbered affiliations see end of article.

Correspondence to

Dr Peter May; mayp2@tcd.ie

\section{ABSTRACT}

Objective To review systematically the evidence on how deinstitutionalisation affects quality of life (QoL) for adults with intellectual disabilities.

Design Systematic review.

Population Adults (aged 18 years and over) with intellectual disabilities.

Interventions A move from residential to community setting.

Primary and secondary outcome measures Studies were eligible if evaluating effect on QoL or life quality, as defined by study authors.

Search We searched MEDLINE, PsycINFO, CENTRAL, CINAHL, EconLit, Embase and Scopus to September 2017 and supplemented this with grey literature searches. We assessed study quality using the Critical Appraisal Skills Programme suite of tools, excluding those judged to be of poor methodological quality.

Results Thirteen studies were included; eight quantitative studies, two qualitative, two mixed methods studies and one case study. There was substantial agreement across quantitative and qualitative studies that a move to community living was associated with improved QoL. QoL for people with any level of intellectual disabilities who move from any type of institutional setting to any type of community setting was increased at up to 1 year postmove (standardised mean difference [SMD] 2.03; 95\% Cl [1.21 to 2.85], five studies, 246 participants) and beyond 1 year postmove (SMD $2.34 .95 \% \mathrm{Cl}$ [0.49 to 4.20], three studies, 160 participants), with total QoL change scores higher at 24 months comparative to 12 months, regardless of $\mathrm{Q} \mathrm{L}$ measure used.

Conclusion Our systematic review demonstrated a consistent pattern that moving to the community was associated with improved QoL compared with the institution. It is recommended that gaps in the evidence base, for example, with regard to growing populations of older people with intellectual disability and complex needs are addressed. PROSPERO registration number CRD42018077406.

\section{INTRODUCTION}

\section{Background/rationale}

The right to live independently in a place of one's own choosing reflects the guiding principles of the 2006 United Nations Convention on the Rights of Persons with Disabilities. ${ }^{1} \mathrm{~A}$ process of 'deinstitutionalisation' - that is,

\section{Strengths and limitations of this study}

- We conducted an extensive systematic search of academic databases, using two reviewers to assess eligibility independently.

- Eligible quantitative and qualitative studies were required to meet a minimum quality threshold.

- We excluded studies not reporting ethical approval, which minimises bias and improves quality standards but potentially excludes earlier studies conducted without reporting guidelines.

- We did not include static cross-sectional studies, requiring that studies evaluated a move in residence for a person with intellectual disability.

- The search strategy is greater than a year old, and further research might be available that would contribute to the review.

moving people with disabilities and mental health problems from institutions to community-living arrangements that support autonomous decision-making and full participation in society - has occurred at different times and different speeds since the 1960s in Scandinavia, the UK, USA, Canada and Australia. ${ }^{2}$

We undertook a systematic review of the evidence on deinstitutionalisation for people with intellectual disabilities (ID). We examined specifically the effect of deinstitutionalisation on economic outcomes and on quality of life (QoL). In this paper we report the results for the QoL studies. The economics results, as well as further details on our search strategy, are available in a companion paper. ${ }^{3}$

QoL is a priority outcome measure for policy-makers but measurement is challenging due to the fluidity of definitions and variability in applications of the concept in practice. ${ }^{45}$ The Schalock framework of QoL is the most widely accepted within the field, with its eight core components of emotional well-being, interpersonal relations, material well-being, personal development, physical well-being, self-determination, social 


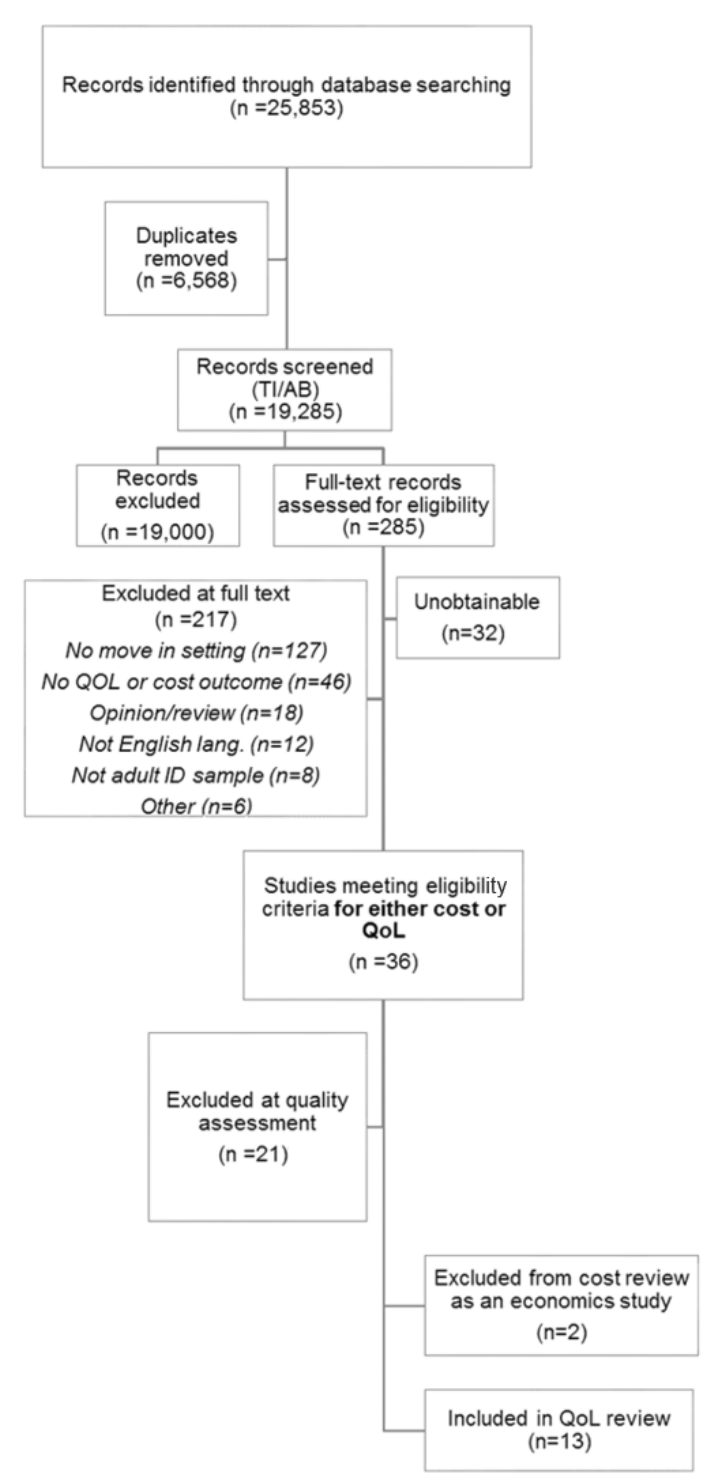

Figure 1 Preferred Reporting Items for Systematic Reviews and Meta-Analyses for QoL search. ID, intellectual disability; QoL, quality of life.

inclusion and rights. ${ }^{6}$ Research to date highlights that people with ID persistently score lower on QoL measures than the general population, ${ }^{7}$ and that level of ID, environmental factors and the level and nature of supports received can impact QoL for people with ID ${ }^{7-9}$ Tracking outcomes, including QoL outcomes, for people with ID following deinstitutionalisation encounters measurement challenges both in the gathering of self-report, proxy and family data and in the value placed on each type of report. ${ }^{6}{ }^{10-15}$ These issues are particularly challenging when engaging people with severe/profound ID yet inclusion of these subgroups is essential. ${ }^{16}$

The impetus for deinstitutionalisation arises from, inter alia, concerns about standards of care, poor outcomes and the recognition that people with ID were being unnecessarily deprived of ordinary lives. ${ }^{17}{ }^{18}$ Research alludes to positive benefits of smaller community-based settings ${ }^{19} 20$ but also attests that gains in health and other outcome measures are not inevitable. ${ }^{19}$ In addition, improvements recorded shortly after a move may plateau after 1 year. ${ }^{21}$ The lack of community readiness to support people to live in the new setting has been proposed as a reason for poor outcomes. The primary focus of policy is on the closure of institutions rather than preparing the community to meet the needs of people with disability now living in the community. ${ }^{22}$ A reduction in the size of setting that the individual moves to cannot be assumed to result automatically in better outcomes in terms of health, wellbeing and overall QoL. This is particularly the case if the new community setting mirrors the culture and practices of the larger institutions with change in how people live, as well as how, when and what type of supports received, being minimal or not materialising. ${ }^{23} 24$

Given the lack of consensus on QoL outcomes as a consequence of deinstitutionalisation there is a need to consolidate the available evidence. This is particularly important in the context of countries that have recently begun or plan to begin implementing a policy of deinstitutionalisation. It is also important for countries that may be challenged by the sustainability and maintenance of the community models put in place in the context of coming demographic change. This is both in terms of the growing older cohort of the general population, which includes the ageing parents and siblings of people with ID, and the increased longevity of people with ID themselves.

\section{Objectives}

To review systematically the evidence on how deinstitutionalisation affects QoL for adults with ID.

\section{METHODS}

\section{Eligibility criteria}

Studies reporting on PICOS (Participants, Interventions, Comparators, Outcomes and Study types) or PEOS (Participants, Exposure, Outcomes and Study types) were eligible for this review. While cross-sectional quantitative studies were generally excluded, as they lacked comparative data on a move, it was not by rule. For example, if a study cross-sectionally asked study participants after a move about changes in QoL arising from that move, this would be included. However, studies that cross-sectionally compared QoL for groups living in institutional and community settings without either group having moved were excluded. Only papers published in English language were eligible.

\section{Types of participants}

Adults (aged 18 years and over) with ID.

\section{Types of intervention/exposure/comparators}

Our intervention of interest was deinstitutionalisationthat is, a residential move from an institutional to a community setting. 
We did not define institutional and community settings ex ante, since no widely accepted definitions (eg, according to the number of residents per unit) exist and we did not want to exclude arbitrarily studies of relevance. Additionally, we were conscious that processes of deinstitutionalisation have happened and are happening at different speeds in different countries, sometimes now involving reinstitutionalisation (moving back from the community to an institution) and transinstitutionalisation (moving between institutions). ${ }^{25}$

Consequently, we assessed the characteristics of institutions and community-living arrangements on the information provided in each paper.

\section{Types of outcomes}

Our prespecified primary outcome of interest was 'QoL' or 'life quality', as defined by study authors. There were no a priori restrictions on the operationalisation of QoL. To be eligible as a primary outcome, we required QoL to be measured both prior to and following a move.

\section{Types of studies/reports}

Study designs eligible for inclusion were: prospective/ retrospective before and after studies, randomised trials, economic evaluations, qualitative/descriptive and exploratory studies.

\section{Search strategy \\ Database search}

To ensure a search strategy that was both sensitive and specific, a comprehensive search methodology to identify both published and grey (eg, policy reports, national/ international guideline documents, etc) literature was developed and executed through routine scientific database searches and grey literature retrieval. Though eligibility was restricted to English language publications, by searching all languages, we were able to identify the extent of potentially eligible additional papers not initially included and assess whether this may have presented a source of possible language bias.

The following electronic databases were searched from date of inception to 11 September 2017: MEDLINE, PsycINFO, CENTRAL, CINAHL, EconLit, Embase and Scopus. Search terms used to guide the review were developed and subsequently finalised by an information specialist (GS) in collaboration with the review team topic experts, and by executing 'scoping' and pilot searches to cross-reference search terms with prior studies and reviews. A combination of title/abstract keywords and related controlled vocabulary terms were incorporated into the search to ensure comprehensiveness. See online supplementary appendix 1 for details. No eligible study looked at both economics and QoL. We reviewed references of included studies and did not identify further eligible studies for inclusion.

\section{Other sources}

The search of grey literature was concerned with non-academic publications, readily available online and included a range of different types of documents such as government, statutory organisation, non-statutory organisation (with particular focus on national disability organisations and university based centres of disability studies) policy, guidance, standards or clinical audit documents which included analytical data-either primary or secondary data analysis. See online supplementary appendix 2 for details.

\section{Study selection and quality assessment}

Screening of titles and abstracts

Two reviewers (RLV and EM) screened the titles and abstracts of retrieved citations, independently, based on the eligibility criteria. Subsequently, approximately 600 conflicts were resolved between these two reviewers on the basis of consensus. Discussions were driven by closely referring to inclusion/exclusion criteria to reach consensus. A key discussion point was verifying that a move had taken place and it was not solely a cross sectional study. In the initial screening stage a particular feature was the inclusion of the concept of adaptation which was viewed through consultation with one of the SR's topic experts not to warrant inclusion as an aspect for QoL. The online reviewer tool COVIDENCE (https://www.covidence. org/) was used to manage the screening process.

\section{Screening of full text reports}

Two independent reviewers (RLV and EM) screened the full texts papers independently, with any conflicts or uncertainties resolved in discussion between the two reviewers.

Assessment of methodological quality/risk of bias

Each included study was assessed for methodological quality using one of a group of standardised instruments developed by the Critical Appraisal Skills Programme (CASP, http://www.casp-uk.net/casp-tools-checklists). The CASP tool because it has been used previously in reviews, and tools have been developed for the varying study designs. Furthermore all CASP checklists cover the three main areas of validity, results and clinical relevance. A pair of reviewers conducted the quality assessment process whereby one reviewer (RLV or EM) assessed the studies' methodological quality and a second reviewer (RLV or EM) performed their own rapid assessment to corroborate quality assessments. Any conflicts were resolved through discussion and consensus. Given that studies of low (or poor) methodological quality can lead to overestimates of the effects of interventions or variables under investigation, and can increase the potential for bias in the results, usually in a positive direction, an a priori decision was made to exclude studies assessed as being of low methodological quality (see online supplementary appendix 3).

Guided by the CASP quality assessment tool, studies involving primary data collection that did not demonstrate evidence of informed consent were excluded. 
Secondary analyses of anonymised data, typically do not require consent as there is no human participation, were not excluded for failing to demonstrate consent agreement.

\section{Data analyses}

Data extraction

Comprehensive data extraction forms were predesigned and piloted to extract relevant data. One reviewer (RLV or EM) extracted the data from the included papers, and a second reviewer (RLV or EM) performed their own rapid assessment of the extracted data to corroborate the accuracy and comprehensiveness of the extracted data. Any conflicts were resolved by discussion and consensus. Relevant data included study design features (randomised trial, prospective or retrospective, etc), study setting (country of origin), participant details (characteristics, numbers, etc), recruitment and sampling, exposure/intervention details, ethical issues (eg, consent), QoL data before and after a move (including summary measures and their $\mathrm{SD}$ as well as qualitative themes) and author-identified implications.

\section{Data syntheses}

\section{Quantitative studies}

We aimed, a priori, to perform a meta-analysis of individual studies' data to achieve an overall (higher level) effect estimate following a move from an institutional setting to a different/community-based setting on QoL. Inclusion in a meta-analysis required sufficient similarity in design (ie, include prospectively collected premove and postmove data) and had to provide overall QoL measures. Specifically they had to have measured QoL prospectively as a pretest (before the move) and post-test (at least one follow-up time point postmove) measure(s). For studies that used repeated post-test measures, we selected QoL measures at one time point for inclusion in the meta-analysis, to avoid over-counting, and described all other time point results narratively. To further reduce characteristic variances in the meta-analyses, we sub-grouped the data according to follow-up at either up to and including 1 year postmove and at more than 1 year following a move from any type of institutional setting to any type of community setting. In addition, while sub-scales of QoL might be chosen as a proxy measure of overall QoL, to be included in the meta-analyses, an overall QoL scale score had to be provided; where sub-scale results only were provided, we present the results for these narratively. High levels of statistical heterogeneity in the analyses were likely due to elements of clinical variation across the included studies (eg, participants with varying levels of ID across studies, and differing age profiles), rather than study design issues. To counterbalance the anticipated subtle differences across the studies (eg, varying degrees of ID/challenging behaviour, etc), we meta-analysed the data using a random-effects model, rather than a fixed-effects. ${ }^{26}$ Lastly, because the instruments used to measure QoL across the included studies differed, we calculated the standardised mean difference (SMD) as per recommended meta-analytical methods. ${ }^{26}$ We interpreted the results as an average of the effect of a move from an institutional setting to a community setting, rather than a 'best-estimate' of the effect, as provided by a fixed-effect model. Studies not meeting these similarity criteria, are reported narratively.

Studies not meeting these similarity criteria, are reported narratively.

\section{QUALITATIVE STUDIES}

We employed a thematic narrative synthesis for identified qualitative studies and the qualitative elements of mixed methods studies. ${ }^{27}$

\section{Patient and public involvement}

The National Disability Authority of Ireland, ${ }^{28}$ an independent state body that advises government and the public sector on policy and practice, contributed to the search strategy.

\section{RESULTS}

\section{Search and selection results}

Database search

The database search for both cost and QoL studies identified 25853 citations for consideration against the eligibility criteria for the review. Following removal of duplicates $(\mathrm{n}=6568), 19000$ citations were excluded on title and abstract, as they clearly did not meet the review's prespecified eligibility criteria (figure 1). A full-text review of the remaining 285 citations was performed, following which a further 217 were excluded and 32 were unobtainable. Reasons for exclusion were: no examination of a change in residential setting (127 articles), no cost or author-defined QoL as an outcome (46), opinion or commentaries and reviews (18), not in English language (12), not an adult population with ID (8) and miscellaneous (6).

Thirty-six articles were therefore identified as meeting the eligibility criteria, of which 21 were subsequently excluded following an assessment of their methodological quality using the CASP tool. Reasons for exclusion at quality assessment included no report of establishing consent of study participants, and insufficient and negligible data on participants and/or outcomes (see online supplementary appendix 3 for a list of studies excluded after quality assessment). Of the 15 studies remaining, two addressed economic outcomes only and are included in a separate paper. ${ }^{3}$ No eligible study looked at both economics and QoL. Thirteen QoL studies passed quality assessment; eight quantitative studies, two qualitative, two mixed methods studies and one case study (online supplementary appendix 4 ).

\section{Grey literature search}

A total of 74 specific reports were identified from the grey literature search. Following detailed review, 30 reports 
were identified as relevant to deinstitutionalisation from a cost and/or QoL perspective. Of these, six include data on premove and postmove measures and so were eligible for this review. Following a quality assessment of each of the six reports that met the eligibility criteria and focused on premove/postmove, none of the reports were included in the final analysis. See online supplementary appendix 2 for details.

\section{Main results}

Description of included studies

Of the 13 included QoL studies, eight were quantitative, ${ }^{29-36}$ two were qualitative, ${ }^{37} 38$ two were mixed methods studies ${ }^{3940}$ and one was a case study. ${ }^{41}$

Characteristics of included studies are summarised in table 1. Sample size ranged from 1 to 76 persons and publication year was from 1994 to 2015. All studies originated from high-income countries, where deinstitutionalisation has been well established in policy and implemented, with six studies originated in Australia, four in the UK, two in Ireland and one in New Zealand. Of the six from Australia, two report different analyses of the same sample and these were dealt with in unison where it was more meaningful to do so.

QoL was operationalised in a range of ways, with some consequent diversity in measurement tools. Three studies used the Life Experiences Checklist (LEC), ${ }^{42}$ a tool which assesses both objective and some more subjective experiences of QoL, and for which validity and reliability data are available. Three studies used the Life Circumstances Questionnaire, a non-standardised tool to assess objective QoL developed by the authors of the studies in which it is used. ${ }^{35}$ Two studies used the QoL Questionnaire (QoLQ), a validated tool providing information on subjective and objective QoL. ${ }^{43}$ Other ways of measuring QoL included aspects of informal social relationships (one study) and family ratings of QoL (one study).

Our quality appraisal assessed risk of bias within studies (online supplementary appendix 4). Of the 13 studies, 12 identified and accounted for important confounding factors. No study was found to have measured exposure or outcome inaccurately, but on these studies we concluded 'can't tell' for seven and three studies respectively.

Five research studies were included which attempted to assess QoL longitudinally, that is, with multiple postmove assessments. Details on follow-up across studies are presented in table 2 .

\section{Key findings}

\section{Quantitative studies}

The key findings of the ten studies with quantitative elements are summarised in table 3 .

Of these, five were deemed suitable for inclusion in a meta-analysis to examine QoL outcomes for people with any level of ID who move from any type of institutional setting to any type of community setting. ${ }^{29} 31$ 33-35 In secondary meta-analyses we performed subgroup analysis by QoL subscale, age and level of ID. In addition, outcomes following a move from one institutional setting to another institutional setting were analysed (two studies)..$^{31} 34$

\section{Overall QoL}

Meta-analysis of QoL outcomes for people with any level of ID who move from any type of institutional setting to any type of community setting are presented in figure 2. QoL was significantly increased at up to 1 year postmove (SMD 2.03; 95\% CI [1.21 to 2.85], five studies, 246 participants, Grading of Recommendations Assessment, Development, and Evaluation (GRADE) level of evidence: moderate) and beyond 1year postmove (SMD 2.34. 95\% CI [0.49 to 4.20], three studies, 160 participants, GRADE level of evidence: moderate), with total QoL change scores higher at 24 months comparative to 12 months.

\section{Level of ID}

Some studies were not disaggregated by level of ID while others provided exact numbers for those with mild, moderate or severe/profound ID. To explore QoL specific to levels of ID, we were able to extrapolate data explicitly on people with mild to moderate ID from four studies, ${ }^{29} 323335$ of which two were suitable for including in a sensitivity analysis (figure 3 ) ${ }^{33}{ }^{36}$ Overall QoL experienced by people with mild/moderate ID did not significantly improve following a move from an institution to any community setting (mean difference (MD) 0.99, $95 \%$ CI [ -0.41 to 0.46$]$, two studies, 51 participants).

One study provided data explicitly on a group of people with severe/profound ID. ${ }^{36}$ These data are also stratified by age $(20-39,40-59,60+)$, but using the average mean and SD scores across the three age groups, results demonstrated significantly increased QoL scores at 24 months postmove in this cohort with severe/profound ID (MD $170.1,95 \%$ CI [ 158.4 to 181.8 ]; $\mathrm{p}<0.0001$ ).

One study assessed QoL in a hospital group $(n=6)$ with mild/moderate ID and severe challenging behaviour (baseline data) prior to a move to community houses and again three and 9 months postmove. ${ }^{32}$ The authors narratively described significant improvements in overall LEC scores (baseline to 3 months, $49 \%$ increase; three to 9 months, additional $24 \%$ increase increase), and in all five LEC domains (between $46 \%$ and 53\%) were described. Domain increases, except Leisure, were maintained 9 months postbaseline $(\mathrm{p}<0.05)$.

One study provided mean LEC change scores stratified by dependency level. ${ }^{29}$ These change scores increased (ie, representing improved QoL) as levels of dependency increased by 11.0 to 13.5 to 17.0 for low, medium and high dependency, respectively, but increases were not statistically significant.

\section{Level of ID and age}

One included study stratified ID by age (20-39, 40-59 and $60+$ ) and by level of ID together (mild/moderate and severe/profound) ${ }^{36}$ As precise numbers in each age category were not provided, results are narratively 




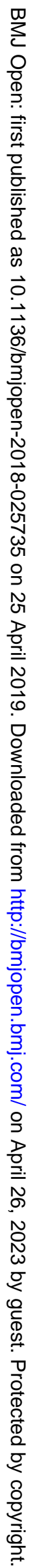









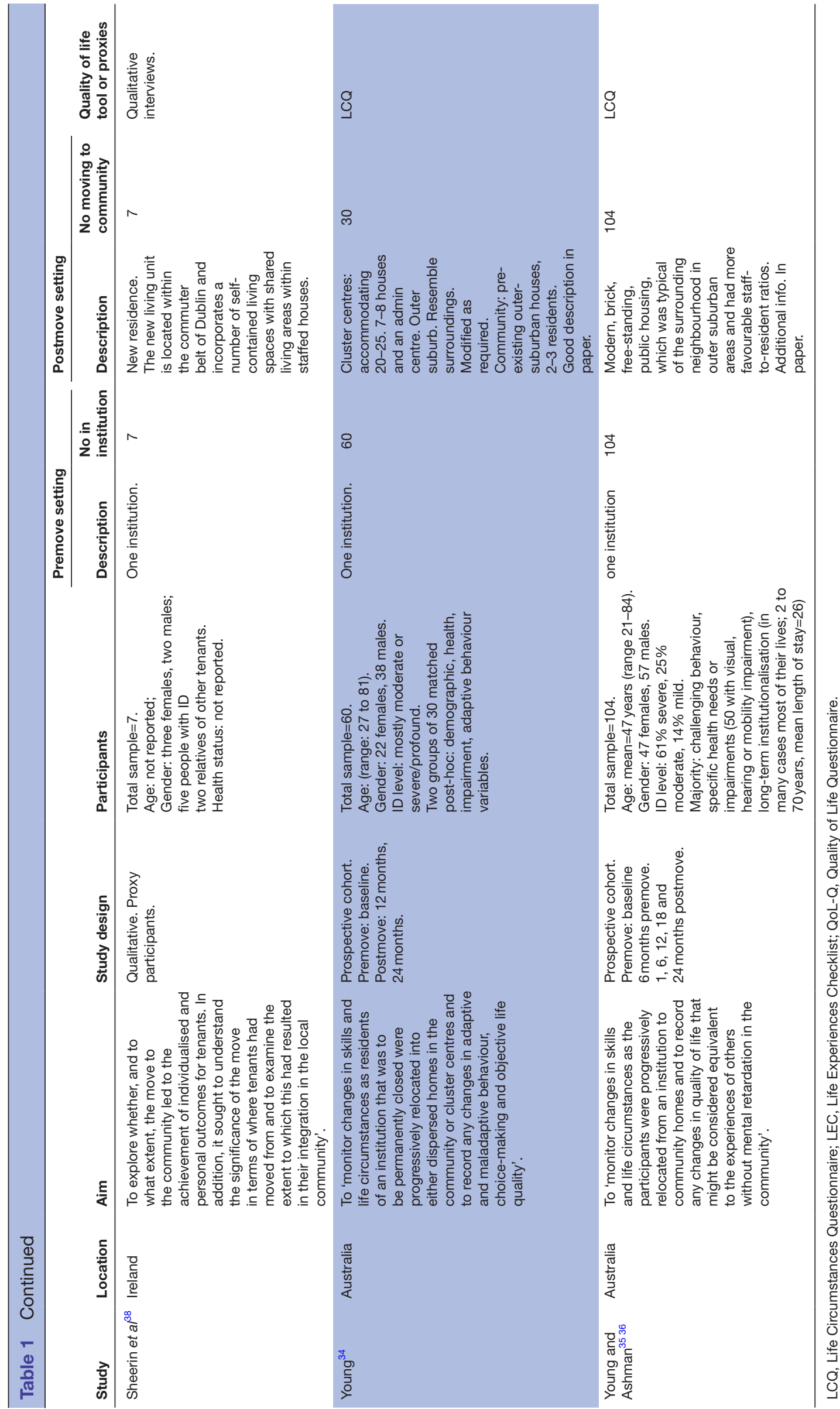


Table 2 Timings of postmove assessments in studies with quantitative quality of life data

\begin{tabular}{|c|c|c|c|c|c|c|c|c|c|}
\hline \multirow[b]{2}{*}{ Study } & \multicolumn{9}{|c|}{ Timing of postmove assessment } \\
\hline & 1 Month & 3 Months & 6 Months & 9 Months & 1 Year & 1.5 Years & 2 Years & 3 Years & 5-9 Years \\
\hline Ager et $a l^{29}$ & & & Yes * & & & & & & \\
\hline Bigby $^{40}$ & & & & & Yes & & & Yes & Yes \\
\hline Cooper and Picton ${ }^{31}$ & & & Yes & & & & & Yes & \\
\hline Howard and Spencer ${ }^{33}$ & & & & & Yes & & & & \\
\hline O'Brien et $\left.a\right|^{39}$ & & & & & & & & & Yes \\
\hline Young $^{34}$ & & & & & Yes & & Yes & & \\
\hline Young and Ashman ${ }^{35} 36$ & Yes & & Yes & & Yes & Yes & Yes & & \\
\hline Total & 2 & 1 & 3 & 1 & 4 & 1 & 2 & 2 & 2 \\
\hline
\end{tabular}

Young and Ashman ${ }^{35}{ }^{36}$ are combined in summary tables, as both papers analyse outcomes for the same cohort at the same time points.

${ }^{*}$ Between 6 and 9 months.

presented. Following a move to the community at 24 months follow-up, people with mild/moderate ID had non-significant $(\mathrm{p}>0.05)$ increases in QoL scores in both the 20-39 and 40-59 age categories, while there were non-significant decreases for those aged $60+$. For people with severe/profound ID, there were statistically significant QoL improvements across all age categories (age 20-39, $\mathrm{p}<0.001 ;$ age $40-59 \mathrm{p}<0.001$, age $60+, \mathrm{p}<0.01)$. Furthermore, participants with severe/profound ID had significantly $(\mathrm{p}<0.01)$ lower total QoL scores than those with mild/moderate ID at both baseline and at follow-up. Participants in all age groups and both levels of ID had significantly increased scores across domains, with the exception of non-significant improvement in physical well-being for the youngest mild/moderate ID group and the oldest severe/profound group.

\section{QoL when moving from institutional setting to institutional setting}

Two studies evaluated QoL following a move from an institution to either another institution or to a clustered setting (figure 4). ${ }^{31}{ }^{34}$ Cluster or campus living refers to specialised housing in an institutional setting or specialised housing for people with disabilities clustered together in an estate/street. This is in contrast to dispersed housing which is non-specialised accommodation spread across a neighbourhood among general population. ${ }^{44}$ Considerable differences in the type of settings the participants moved to precluded combination in a meta-analysis.

Overall QoL-Q scores, at both 6 months and 3 years postmove, improved significantly for a sub-group of 19 who moved to refurbished units in a different institution. ${ }^{31}$ A sub-group of individuals (with challenging behaviour), who moved from institutions to cluster centres (accommodating between 20 and25 residents in each centre) had significantly higher QoL scores at 12 (MD 97.8, 95\% CI [68.16 to 127.44]) and 24 months (MD 103.5, 95\% CI [75.77 to 131.23$]$ ), postmove. ${ }^{34}$ All QoL sub-domains improved significantly with a linear trend from premove to 12 and 24 months postmove to cluster centres (all $\mathrm{p}<0.001){ }^{34}$

Direct comparison of two alternative settings demonstrated that individuals who moved from institutions to dispersed small group community homes had significantly higher QoL scores at 12 (MD 26.9, 95\% CI [1.27 to 52.53]) and 24 months (MD 39.2, 95\% CI [14.31 to 64.09]), postmove compared with clustered settings (figure 5) ${ }^{34}$ When subdomain outcomes were compared between dispersed community and clustered settings over time, dispersed settings afforded significantly better physical well-being $(p<0.005)$, community access $(p=0.001)$, routines $(p<0.01)$, self-determination $(p<0.01)$, residential well-being $(\mathrm{p}<0.01)$ and general life improvements $(\mathrm{p}<0.001)$. Groups did not differ on material well-being and social/emotional well-being.

\section{QUALITATIVE STUDIES}

The main themes identified in the five qualitative or mixed methods studies were: 1) positive changes experienced following the move to the community and a sense of 'freedom' and independence living in the community increased QoL; 2) compatibility among housemates; 3) perceived staff's role in supporting community living; 4) social integration and family contact; 5) ongoing challenges for individuals' QoL. Key qualitative findings are presented in table 4.

\section{A sense of 'freedom' and independence living in the community increased QoL}

Positive outcomes for individuals' well-being following a move to the community were reported in all five studies. In contrast to the experience of living in an institutional setting, individuals' new living arrangement in the community was perceived as a more suitable environment as it was more private, less noisy with more 


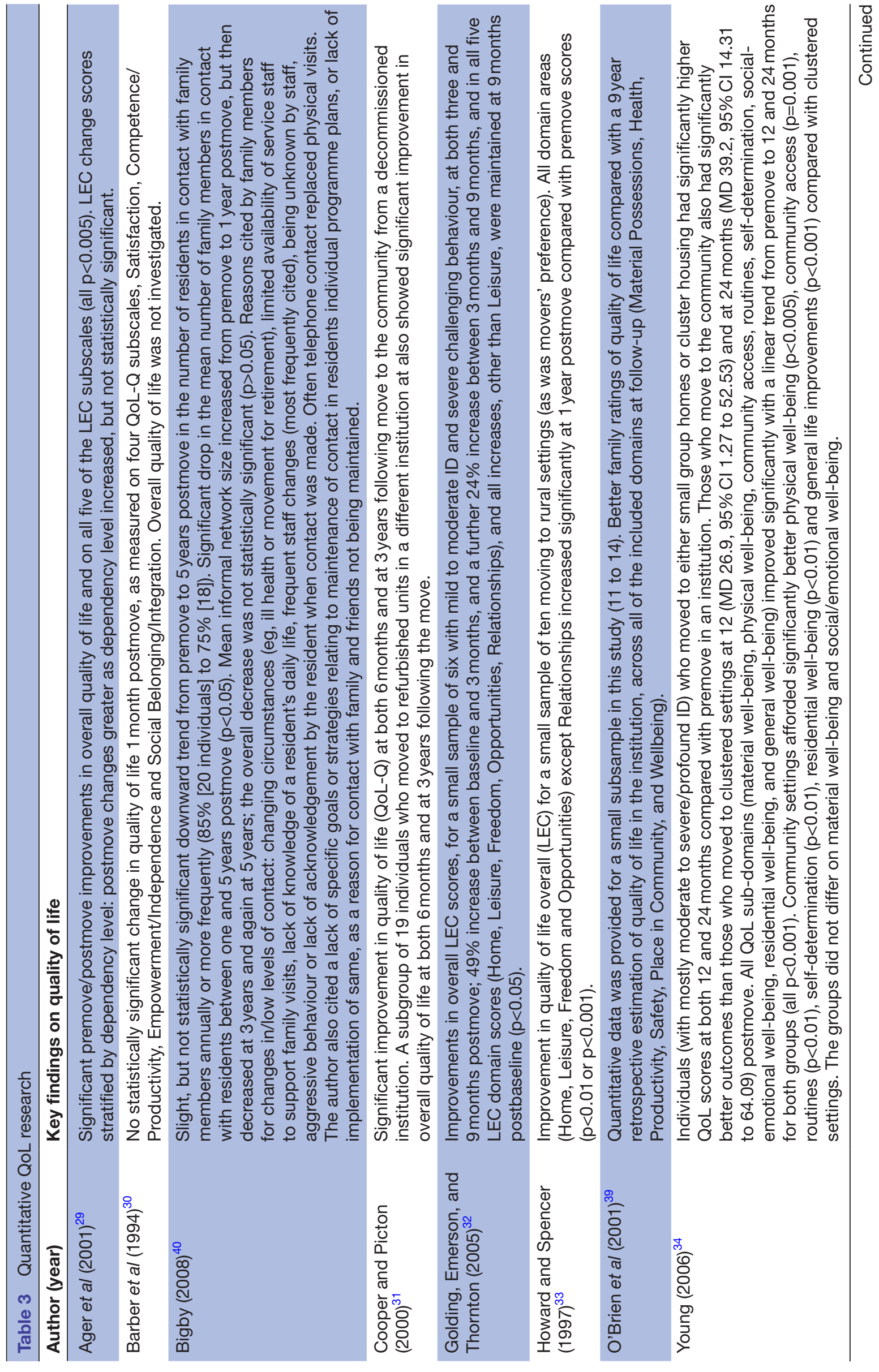






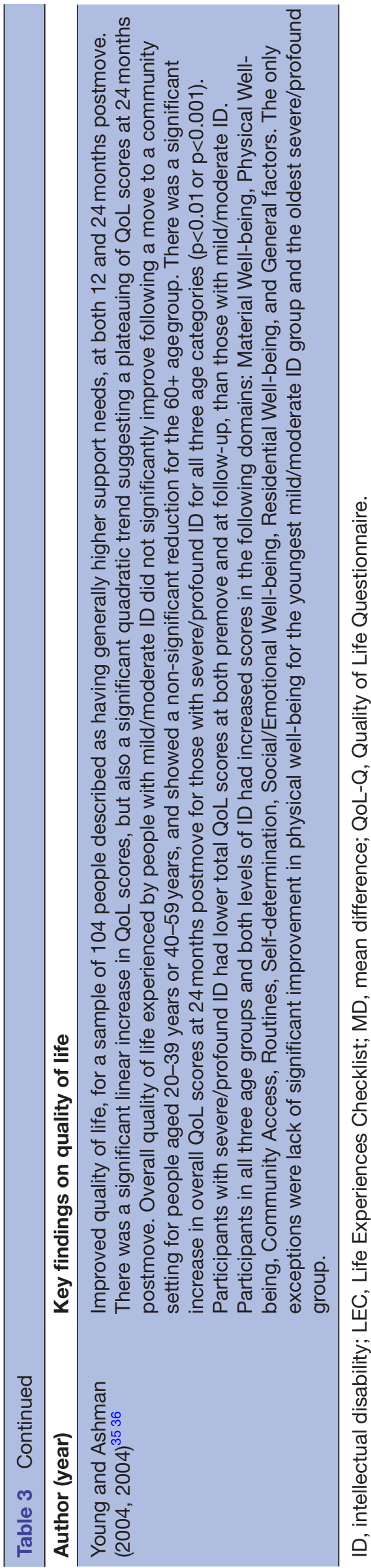

space including a garden area and wheelchair access. ${ }^{37} 38$ Increased independence regarding money management gave participants the freedom to make every day personal choices that positively impacted their QoL. ${ }^{38}$ Compared with their previous experience living in a more restricted residential environment, moving to the community for all participants in three studies was perceived as giving them a sense of 'freedom' ${ }^{37-39}$ Moving to the community was also connected with increased personal space and privacy resulting in improved QoL.

Considering compatibility among housemates increased QoL

More careful consideration of the impact of individuals' compatibility with housemates when placing individuals in the community houses is reported as positively impacting individuals' QoL. ${ }^{37}{ }^{39}$ In one study, individuals were perceived by proxies to have been previously affected by housemates making noise or engaging in self-injurious behaviour and indicated the importance of housemate compatibility to QoL. ${ }^{37}$

\section{Perceived staff roles in supporting community living}

Staff's support roles were perceived as contributing to individuals' QoL. ${ }^{37}{ }^{38}$ Permanent staff familiar with individuals' interests and choices helped improve individuals' participation in the community and alleviated some individuals' stress related to staff turnover. ${ }^{37}{ }^{38}$ However, some other participants had higher expectations of staff support and involvement, which subsequently negatively impacted their perceived QoL. ${ }^{38}$

\section{Social integration and family contact}

The impact of the move on the individuals' social integration and family contact as it related to their QoL was a common theme in all five studies. The case study presents the life history of a woman with learning disabilities and severe challenging behaviour who after 30 years in UK institutions, experienced increases in QoL following her eventual move to a small community staffed house. ${ }^{41}$ In particular, access to individualised day programmes increased perceived positive social integration. Additionally, increased contact with her family due to the community home's significantly closer proximity to her family meant she ultimately could get to know her siblings after years of separation, and visit her family more regularly. This increased integration into her family's life had a perceived positive impact on her QoL, as noted especially by her mother.

An Australian mixed methods study specifically focused on the significance of the role of informal social networks on QoL. Four types of informal networks for residents were identified: (1) non-existent ( $\mathrm{n}=4$ participants); (2) special occasion family $(n=6)$; (3) engaged family $(n=9)$; (4) friendship-based $(n=5)$. Although one of the community house staff's key responsibilities was to support residents maintain contact with family and friends following relocation, this was not substantiated in residents' individual plans. ${ }^{40}$ 


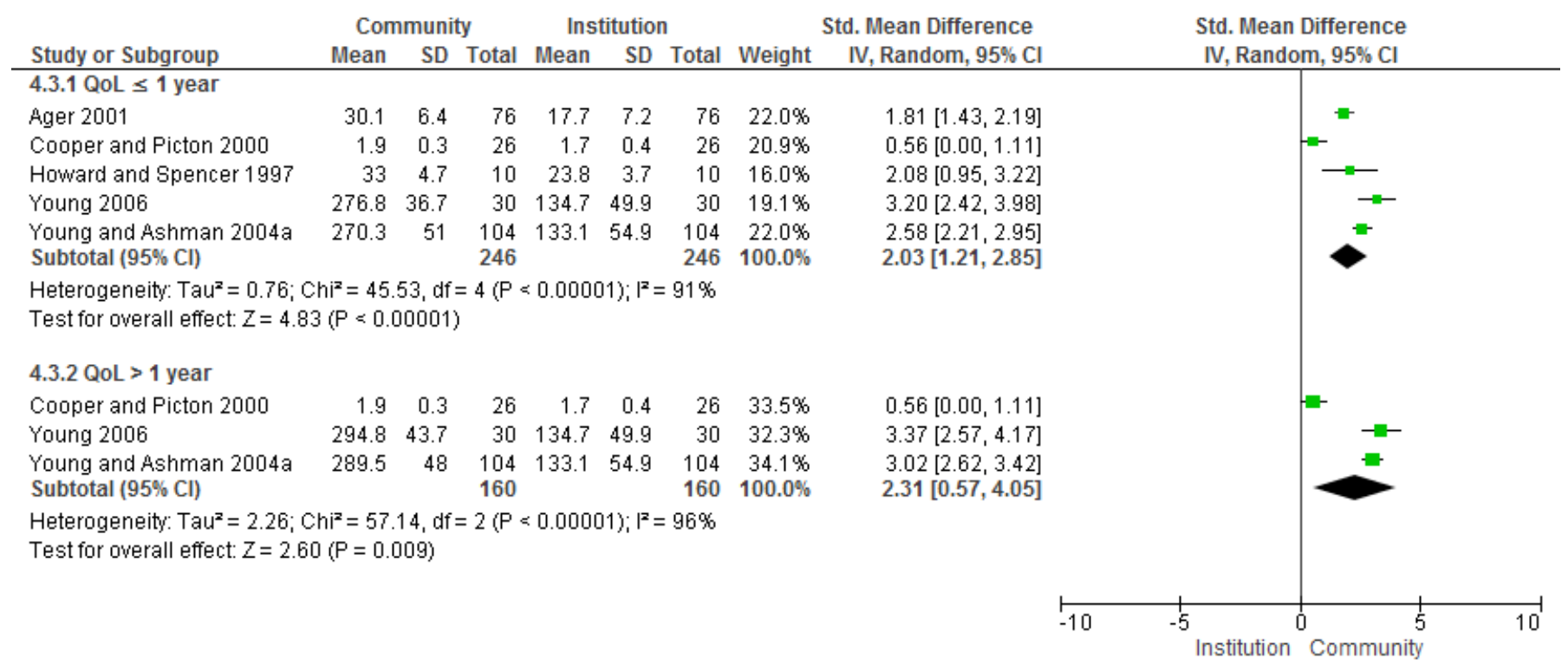

Figure 2 Quality of life with any level of intellectual disability postmove from any institutional setting to any community setting.

In another study, it was perceived that all participants were accessing more services within the community and also 'getting out into the community' more as a result of the move. ${ }^{37}$ However, the individuals with ID were not necessarily more integrated with people in the community, and instead showed a preference for being with people with whom they were more familiar (from the community house). In another study, relatives' experiences differed on how socially integrated into the community their relatives with ID were, ranging from those who felt their relative was welcomed to others who perceived they were not. ${ }^{38}$ Overall, most of the participants in this study indicated that they did not feel integrated into the local community and stated that they did not know anyone there. Indeed, some participants appeared to be even more isolated than they were when living in their previous residential setting.

\section{Ongoing challenges for individuals' QoL}

Although all five studies with a qualitative component reported positive outcomes for individuals with ID moving into the community, ongoing challenges to individuals' QoL were also reported. Adjustment to the move could reportedly take months, depending on the individuals' transition circumstances. Ongoing difficulties included day programmes being too cramped, with poor consideration of the individuals' needs in particular in relation to challenging behaviours; unavailability of speech and language therapy or communication aids ${ }^{37}$; family contact was infrequent and accessing amenities was inconvenient due to a postmove rural location ${ }^{37}$; lack of adequate funding meant reduced night time community staffing and no overnight trips ${ }^{37}$; and some participants experienced a loss of security following the move related to change in staffing routines, leading to loneliness and insecurity. ${ }^{38}$

A summary of the main findings from this review is presented in table 5 .

\section{DISCUSSION}

\section{Key findings}

Our systematic review yielded quantitative and qualitative findings that deinstitutionalisation is associated with QoL improvements for people with ID. These findings are broadly consistent with prior reviews. ${ }^{23} 45-47$

There was substantial agreement across quantitative analysis regarding improved QoL which held for shorter (up to 1 year) and longer (more than 1 year) term QoL measures, with a slightly increased difference between premove and longer term QoL (overall) than shorter-term QoL. This challenges to some extent previous findings which indicated modest gains which occurred

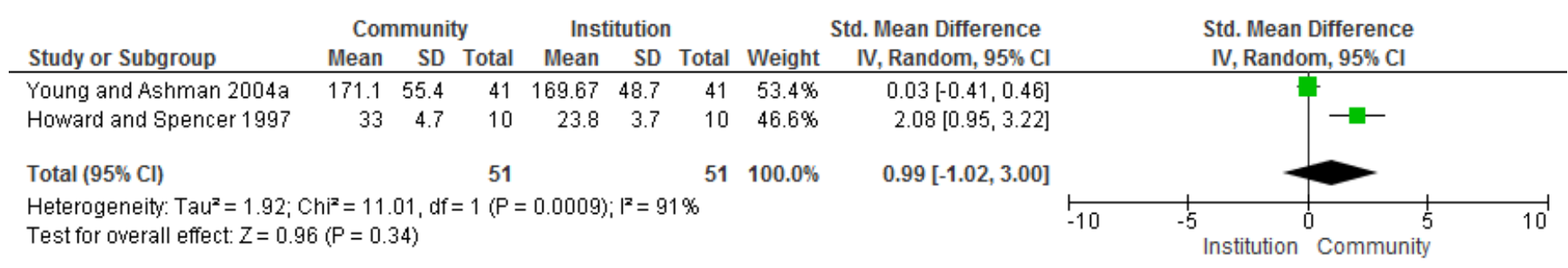

Figure 3 Quality of life in people with mild/moderate intellectual disability only postmove. 


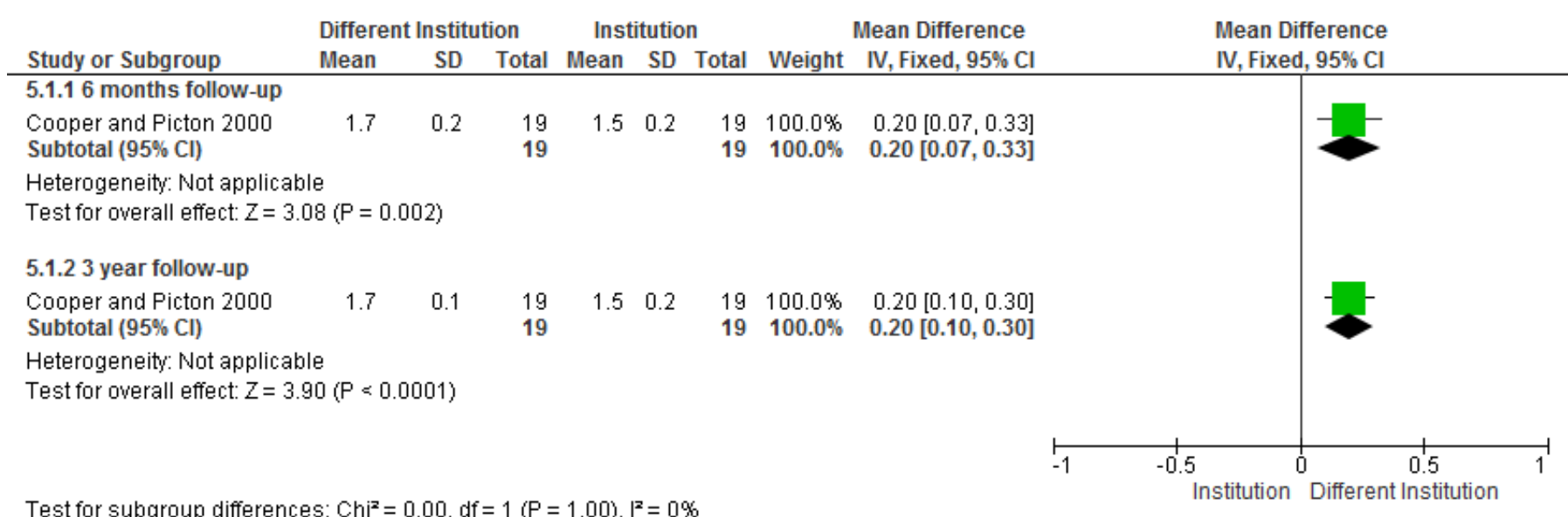

Figure 4 Quality of life following move from one institution to a different institution.

soon after the move and plateaued at 1 year, with these studies showing continued gains after 1 year. ${ }^{48}$

When institutional settings close, it tends to happen in a phased approach with evidence showing the younger less complex needs cohort moving first. ${ }^{19} 49$ The present analysis highlighted the positive gains in QoL that can be experienced by people with severe/profound ID and higher support needs. This finding also held for most aspects or sub-domains of QoL where these were studied.

Qualitative studies found that movement to community residences facilitated an improved sense of wellbeing, freedom and independent decision-making. When housemate compatibility was more carefully considered prior to their move, individuals had higher quality daily living experiences. There remain, however, challenges for aspects of QoL, including social integration and relationships, and physical well-being for certain subgroups.

Becoming part of the community is considered one of the main advantages associated with living in the community. ${ }^{404}$ In our review, mixed findings are reported on the impact of the move on individuals' social integration into the wider community. Authentic community participation eluded many individuals and some individuals reported feeling lonelier since the move due to differing expectations of staff supports. This concurs with previous work with regard to the importance of the quality of supports provided and further highlights that an improvement in QoL is not inevitable but must be managed and supported. ${ }^{40}$ Prior to the move, individuals living in institutional settings had relied more heavily on staff to care for their basic living needs. Following the move to the community with an increased emphasis on nurturing independence, some individuals may experience a loss of security. Without the support from staff to maintain family contact and retain friendships from previous residential setting, individuals' sense of disconnectedness could be compounded. It would be interesting in future research to see if this disconnect is better bridged over time.

This review indicates that support from staff to facilitate integration into the community while maintaining family and other social contacts is vital to the individuals' QoL. Individual transition-planning requires thoughtful consideration to address the issue of housemate compatibility, and service user expectations about the level of support provided by staff. Increased contact with family could create new opportunities for family to participate more in supporting social activities (eg, overnight trips and excursions) that could otherwise be restricted due

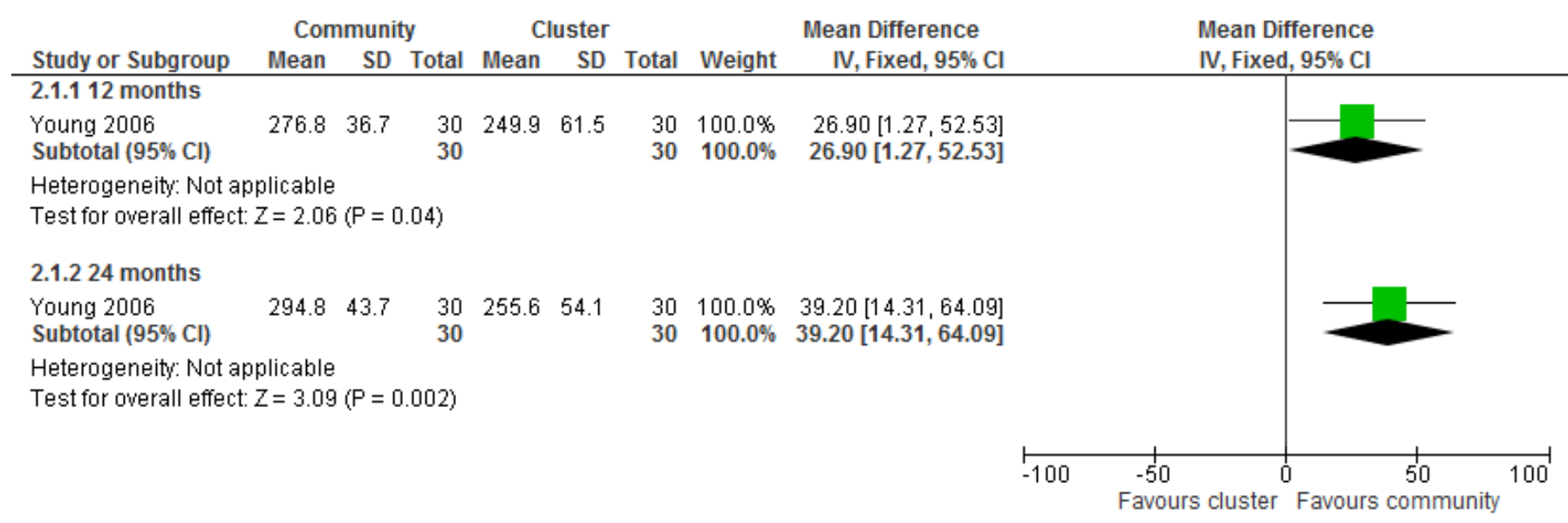

Figure 5 Quality of life in community versus cluster settings following a move from an institution. 


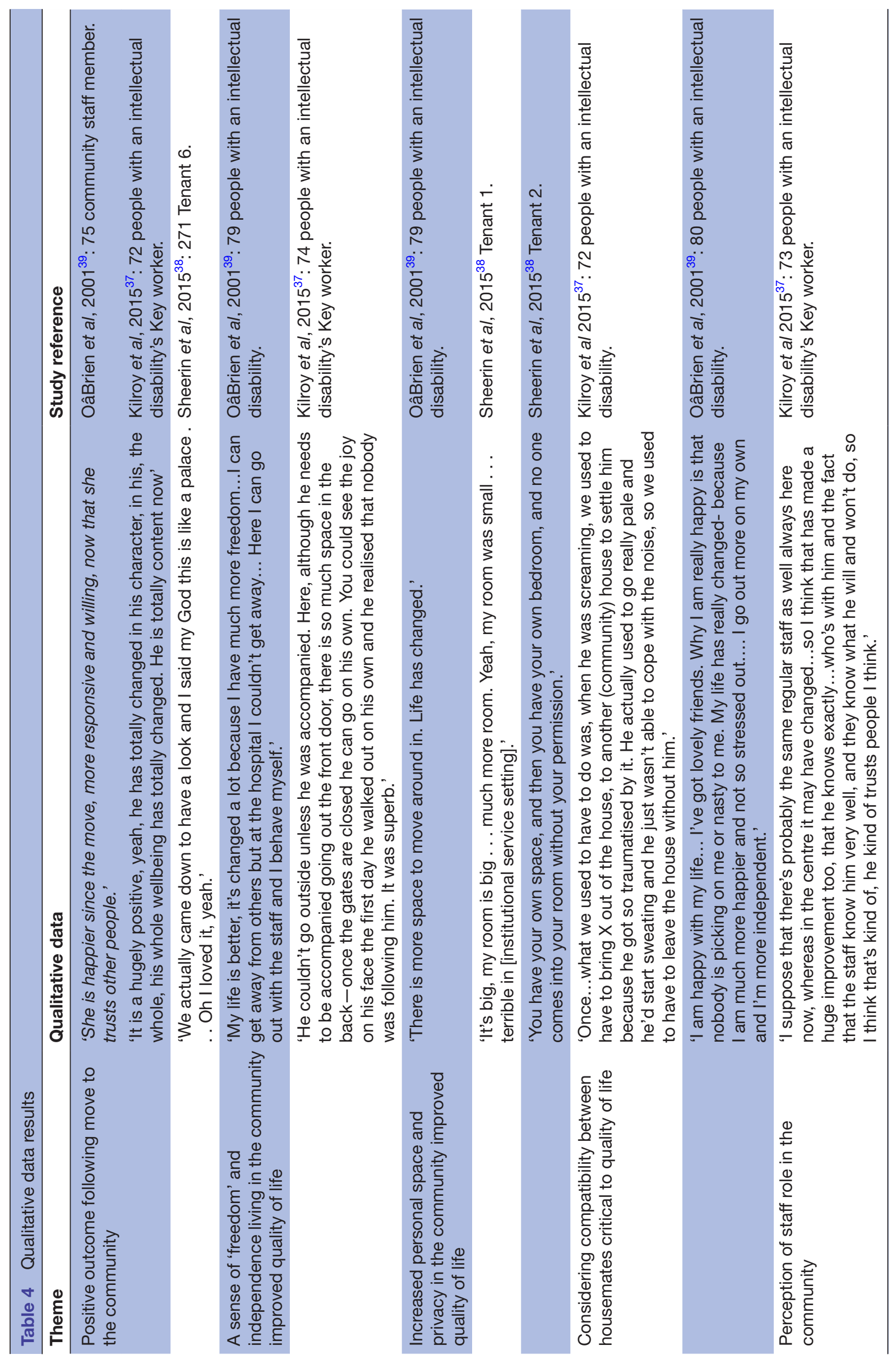

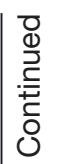

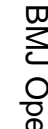






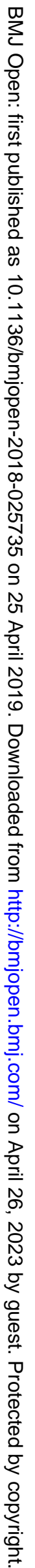


Table 5 Summary of findings: premove compared with postmove for quality of life in persons with any level of ID and any setting

\section{Patient or population: Quality of life \\ Setting: Institutional and Community Intervention:Postmove \\ Comparison: Premove}

\begin{tabular}{lcll}
\hline & $\begin{array}{l}\text { No of participants } \\
\text { (studies) }\end{array}$ & $\begin{array}{l}\text { Certainty of the } \\
\text { evidence (GRADE) }\end{array}$ & Comments \\
\hline $\begin{array}{l}\text { Quality of Life: } \leq 1 \text { year } \\
\text { postmove }\end{array}$ & 246 (5 observational studies) & $\begin{array}{l}\oplus \oplus \oplus \bigcirc \\
\text { Moderate a b }\end{array}$ & $\begin{array}{l}\text { a. Observational (pre/post) studies } \\
\text { b. Statistical heterogeneity }\end{array}$ \\
$\begin{array}{l}\text { Quality of Life: }>1 \text { year } \\
\text { postmove }\end{array}$ & 160 (3 observational studies) & $\begin{array}{l}\oplus \oplus \oplus \bigcirc \text { Moderate }^{\text {a,b }} \\
\text { a. Observational (pre/post) studies } \\
\text { b. Statistical heterogeneity }\end{array}$ \\
\hline
\end{tabular}

GRADE working group grades of evidence.

High certainty: We are very confident that the true effect lies close to that of the estimated effect.

Moderate certainty: We are moderately confident in the effect estimate: The true effect is likely to be close to the estimated effect, but there is a possibility that it is different.

Low certainty: Our confidence in the effect estimate is limited: The true effect may be substantially different from the estimate of the effect. Very low certainty: We have very little confidence in the effect estimate: The true effect is likely to be substantially different from the estimate of effect.

GRADE, Grading of Recommendations Assessment, Development, and Evaluation; ID, intellectual disability.

to limited funding. Yet, despite the ostensible QoL benefits of family contact and relationships, and that community living might facilitate same, there is evidence in the findings that social network sizes may not increase significantly in the longer term following a move, and that family contact in fact shows a downwards trend.

\section{Strengths and limitations}

This study has followed best practice guidelines in systematic evidence reviews where possible. A search strategy was devised following pilot searches and multiple meetings of a team that includes subject experts in ID, an information specialist and a systematic review specialist. The breadth and thoroughness of the search strategy was illustrated in a very large number (over 25000 ) of returned titles and abstracts from databases, and each of these was independently reviewed by two team members. Likewise, all full texts accessed were independently reviewed by two team members. For studies included in the review, quality assessment and data extraction was performed by one reviewer with a corroborating rapid review by a second reviewer. It should be noted that all included studies originated from high-income countries, where deinstitutionalisation has been well established and implemented, and thus generalisability of the findings for low-to-middle-income countries is not clear due to local cultural challenges to implementation. However, the broad findings on enablers to de-institutionalisation in improving QoL, particularly those garnered from the qualitative studies, should provide useful indicators for implementation.

Nevertheless, there are a number of important limitations to our work. We were unable to define ex ante definitions of 'congregated/institutional' and 'community' settings. In practice, institutions were clearly institutions-places with a number of institutional features, and described as such. Community definitions were more nebulous and we made the best judgements we could as well as providing all available information on the precise conditions in each study, to allow for third party evaluation. We are satisfied retrospectively with this approach. Applying a hard definition would have been very problematic, due to reporting insufficiencies of the extant research. In devising our search strategy we were faced with profound challenges in defining our intervention. While every effort was made to include all potentially relevant terms, as the high number of reviewed titles and abstracts testifies, it is possible that we overlooked some terms that would have captured other relevant material.

Similarly, QoL is a multi-faceted concept with many potential definitions. We considered different approaches to capturing QoL, for example, including all identified sub-domains in the Schalock framework,${ }^{6}$ but we did not consider it feasible to identify reliably all named domains and their synonyms. We therefore chose author-defined QoL as our outcome of interest.

In reviewing returned studies from the database search, we used two independent reviewers for title/abstract and full texts, but one reviewer at quality assessment and data extraction with a second reviewer providing a corroborating review. While corroboration by a second reviewer can be acceptable in the review process, the lack of independent second reviewer assessments does introduce the potential for bias in the quality assessment and data extraction phases of the review. Thirty-two $(17 \%)$ of the studies that we identified as suitable for full text review proved unobtainable and so are not included in our final analyses, thus, potentially introducing selection bias. These studies, however, are on average older than those we were able to access and are listed in online supplementary appendix 5 . 
The decision to require documentation of consent obtained from participants with ID and ethical considerations did mean that a number of older studies were excluded as well as all of the grey literature. We considered that categorically requiring reporting of a consent process helped to safeguard against: (a) bias derived from inappropriately conducted research (eg, acquiescence), and (b) inclusion of research with inadequate ethical protocols in meta-analyses and consequent publication of new and original research findings based partly on such research. In consideration of the importance of choice and subjective evaluation, and the potential for conflict of interest, we viewed this as an unacceptable risk of bias. However, we are not implying that good or appropriate ethical practice was not adhered to in excluded studies, merely that we could not necessarily ascertain this. The clear majority of research excluded for reasons of ethical considerations also had other methodological shortcomings that would have been sufficient to exclude the study from our review, either in concert with the ethical considerations, or in and of themselves.

Included studies were all observational and had a sample size range of 1 to 76 . It is not surprising that observational designs dominate in this field and to maximise confidence in our results we ensured that all included studies met a minimum threshold for methodological quality using the CASP quality assessment tool (that is 'good/ high' quality). Additionally to assess the level and quality of the evidence for QoL, we performed a GRADE assessment of the summary results. GRADE provides a system for rating the quality of the evidence, based on a collective assessment of study design, risk of bias, imprecision, inconsistency, indirectness and magnitude of effect, on the results of meta-analysed data. For both QoL measures, that is up to 1 year postmove, and more than 1 year postmove, the quality of evidence is moderate (downgraded due to observational study designs and statistical heterogeneity) indicating moderate confidence that the average effect estimates are reflective of 'true' estimates, and that the addition of further studies is unlikely to substantially change these results (table 5).

Acknowledging the challenges in measurement and reporting of QoL by proxy, particularly for people with severe/profound ID, the analysis used a random effects rather than a fixed effects model, to counterbalance any potential subtle differences across studies with regard level of ID and type of reporting. Future studies could explore the differences in type and change in proxies over time and the impact on QoL measurement. We note the high levels of heterogeneity in the synthesised results for QoL. This, we believe, is likely to be explained by both clinical and methodological variation within the included studies. While we attempted to explore this further through sub-groups analyses, we highlight that it needs to be considered when interpreting the results of the review.

We also included only English language studies in our review, excluding 12 studies on this basis, which is another potential source of bias. These studies are listed in online supplementary appendix 6 and were variously published in French (7), Croatian (2), German (2) and Japanese (1). It was therefore notable that no studies either included in the review or excluded due to language considerations originated in the Nordic countries with the longest history of deinstitutionalisation. It is possible that researchers and/or government agencies in these countries evaluated the impact of deinstitutionalisation prior to the mass uptake of online publishing, and that these evaluations exist somewhere purely offline.

The grey literature search was conducted by topic experts on the websites of research centres active in this field and those of governments in countries at the forefront of deinstitutionalisation in ID. This may have biased reviewed studies against other nations and research groups. While much grey literature was excluded from the review for considerations including lack of comprehensive reporting on ethics, there may be findings of import within that literature that may warrant separate review or discussion.

\section{Future research}

Subpopulations with additional needs or who require high-levels of support have received insufficient attention in the literature, and research of high methodological quality is required to better understand the needs of a range of groups. It could be reasonably concluded from the available evidence that a move to the community provides similar benefits for people with more severe levels of ID and that people with high-support needs or challenging behaviour experience similar benefits to their counterparts who have fewer additional needs. This conclusion is based on a few studies and is subject to limitations similar to the wider literature.

With people with ID now living much longer into old age than previous generations, how older age interacts with residential moves also needs comprehensive investigation. Physical well-being has emerged as an aspect of QoL which may not improve as much for groups encompassing younger people with mild ID and older people with severe ID. While it is possible that younger groups reach a relative ceiling of functioning and well-being, with little room for additional improvement per se, older adults with ID may require additional and different supports. Special attention must be paid to the population with dementia, a population which likely faces additional and growing challenges and may require specific supports for optimal QoL. Research is also lacking on people with other specific health needs or impairments (eg, those using ventilators), those who present a forensic risk and ex-prisoners. We have limited information about whether and how these particular groups' QoL might be affected by where they live, and furthermore how such clients might ultimately be best supported to experience the benefits of community living and optimal QoL.

There is a scarcity of comprehensive data on outcomes more than 2years postrelocation to the community. Existing evidence indicates that while QoL may increase 
following a move to a non-institutional setting, it begins to plateau between one and 2 years after the move. Longitudinal studies with longer follow-up periods are warranted to monitor whether the improvement of outcomes is maintained at least in the longer term. Again, serious attention must be paid to the different populations outlined above and to understanding the mechanisms by which changes or improvements in QoL occur, including the impact of changes in services available, proximity to important services and opportunities.

\section{CONCLUSION}

There was a substantial level of agreement between quantitative meta-analytic (ie, SMDs for all movers) and other results, supported by the qualitative findings, that a move to the community was associated with improved QoL compared with the institution. Qualitative studies in particular suggest that observed improvements occur through improved well-being, freedom and independent decision-making, more careful consideration of housemate compatibility, increased family contact and social integration opportunities.

While it is tempting to suggest sufficient evidence exists, there remain a number of unanswered questions. There is not yet enough knowledge about the long-term course of QoL outcomes, which is of particular interest considering the ageing nature of this population, or for specific aspects of QoL, including social integration and relationships. Subpopulations with additional needs or who require high-levels of support have received insufficient attention in the literature, and research of high methodological quality is required to better understand heterogeneity of need and outcome. Moreover, qualitative studies highlighted a number of negative QoL outcomes including insecurity, fear and loneliness that emphasise that gains do not come without a cost. These concerns also need further investigation.

Future research must address these issues to ensure that, as deinstitutionalisation continues around the world in the context of profound demographic change, people with ID are supported to live healthy, independent lives of their own choosing.

Author statement

\section{Author affiliations}

${ }^{1}$ Dean of the Faculty of Health Sciences, Trinity College Dublin, Dublin, Ireland

${ }^{2}$ IDS TILDA, University of Dublin Trinity College, Dublin, Ireland

${ }^{3}$ School of Nursing and Midwifery, University of Dublin Trinity College, Dublin, Ireland ${ }^{4}$ Centre for Health Policy and Management, Trinity College Dublin, Dublin, Ireland

${ }^{5}$ TILDA, University of Dublin Trinity College, Dublin, Ireland

${ }^{6}$ National Learning Network, Institute of Technology Blanchardstown, Dublin, Ireland ${ }^{7}$ Faculty of Health Sciences, University of Dublin Trinity College, Dublin, Ireland

${ }^{8}$ College of Public Health, Temple University, Philadelphia, Pennsylvania, USA

${ }^{9}$ Faculty of Health Sciences, University of Sydney, Sydney, New South Wales, Australia

${ }^{10}$ Cicely Saunders Institute, King's College London School of Medical Education, London, London, UK

${ }^{11}$ Trinity Centre for People with Intellectual Disabilities, School of Education, University of Dublin Trinity College, Dublin, Ireland
Contributors MMC and PMC co-designed the original review protocol, oversaw all phases of the review process, and drafted and revised the paper. MMC is the guarantor. RLV and EM were lead researchers on all stages of the systematic review (title and abstract, full text, quality assessment, data analysis) and led authorship of the manuscript. PM co-designed the original review protocol, project-managed the review process and, drafted and revised the paper. NW conducted the grey literature search, and drafted and revised the paper. GS was the information specialist, co-designing and running the database searchers, and revising the paper. RS co-designed the original review protocol, advised and contributed throughout the review process as a topic expert, and drafted and revised the paper. VS co-designed the original review protocol, advised and contributed throughout the review process as a systematic review expert, and drafted and revised the paper. CN co-designed the original review protocol, advised and contributed throughout the review process as an economics expert, and drafted and revised the paper. M-AOD co-designed the original review protocol, led the grey literature search, advised and contributed throughout the review process as a topic expert, and drafted and revised the paper.

Funding The study was funded by the Department of Health (Ireland), with commissioning assistance by the Health Research Board (Ireland).

Competing interests None declared.

Patient consent for publication Not required.

Provenance and peer review Not commissioned; externally peer reviewed.

Data sharing statement There are no unpublished data from this study. This is a systematic review that generates no new data. We make our results available in full in the manuscript. Further questions should be directed to the corresponding author.

Open access This is an open access article distributed in accordance with the Creative Commons Attribution Non Commercial (CC BY-NC 4.0) license, which permits others to distribute, remix, adapt, build upon this work non-commercially, and license their derivative works on different terms, provided the original work is properly cited, appropriate credit is given, any changes made indicated, and the use is non-commercial. See: http://creativecommons.org/licenses/by-nc/4.0/.

\section{REFERENCES}

1. Nations U. Convention on the rights of persons with disabilities. New York, 2006.

2. Mansell J, Beadle-Brown J. Deinstitutionalisation and community living: position statement of the comparative policy and practice special interest research group of the international association for the scientific study of intellectual disabilities. J Intellect Disabil Res 2010;54:104-12.

3. May P, Lombard-Vance R, Murphy E, et al. The economic effects of deinstitutionalisation for adults with intellectual disabilities: a systematic review, 2018.

4. Sexton E, O'Donovan M-A, Mulryan N, et al. Whose quality of life? A comparison of measures of self-determination and emotional wellbeing in research with older adults with and without intellectual disability. J Intellect Dev Disabil 2016;41:324-37.

5. Lee Mclntyre L, Kraemer BR, Blacher J, et al. Quality of life for young adults with severe intellectual disability: mothers' thoughts and reflections. J Intellect Dev Disabil 2004;29:131-46.

6. Schalock RL, Brown I, Brown R, et al. Conceptualization, measurement, and application of quality of life for persons with intellectual disabilities: report of an international panel of experts. Ment Retard 2002;40:457-70.

7. Schalock RL. The concept of quality of life: what we know and do not know. J Intellect Disabil Res 2004;48:203-16.

8. Perry J, Felce D. Correlation between subjective and objective measures of outcome in staffed community housing. J Intellect Disabil Res 2005;49:278-87.

9. Totsika V, Felce D, Kerr M, et al. Behavior problems, psychiatric symptoms, and quality of life for older adults with intellectual disability with and without autism. J Autism Dev Disord 2010;40:1171-8.

10. Stancliffe RJ. Proxy respondents and the reliability of the quality of life questionnaire empowerment factor. J Intellect Disabil Res 1999;43:185-93.

11. Simões $C$, Santos $S$. The quality of life perceptions of people with intellectual disability and their proxies. $J$ Intellect Dev Disabil 2016;41:311-23.

12. Stancliffe RJ. Proxy respondents and quality of life. Eval Program Plann 2000;23:89-93. 
13. Kane RA. Definition, measurement, and correlates of quality of life in nursing homes: toward a reasonable practice, research, and policy agenda. Gerontologist 2003;43:28-36.

14. Lesa $\mathrm{H}$, Janet $\mathrm{M}$, Denise $\mathrm{P}$, et al. Assessing family outcomes: psychometric evaluation of the beach center family quality of life scale. Journal of Marriage and Family 2006;68:1069-83.

15. Katja P, Bea M, Carla V. Domains of quality of life of people with profound multiple disabilities: the perspective of parents and direct support staff. $J$ of Applied Research in Intellectual Disabilities 2005;18:35-46.

16. Townsend-White C, Pham AN, Vassos MV. Review: a systematic review of quality of life measures for people with intellectual disabilities and challenging behaviours. $J$ Intellect Disabil Res 2012;56:270-84.

17. School of Social Work and Social Policy, La Trobe University. In: Bigby C, Fyffe C, Mansell J, eds. From ideology to reality: current issues in implementation of intellectual disability policy. Bundoora, Victoria: Roundtable on intellectual disability policy, 2006.

18. McCallion P, Jokinen N, Aging JMP, et al. A comprehensive guide to intellectual and developmental disabilities. eds. Baltimore: Paul Brookes Press, 2017.

19. Emerson E, Hatton C. Deinstitutionalization in the UK and Ireland: outcomes for service users. J Intellect Dev Disabil 1996;21:17-37.

20. Mansell J. Deinstitutionalisation and community living: progress, problems and priorities. J Intellect Dev Disabil 2006;31:65-76.

21. Monali $\mathrm{C}, \mathrm{Bb}$ A. Deinstitutionalization and quality of life of individuals with intellectual disability: a review of the international literature. $J$ of Policy and Practice in Intellectual Disabilities 2011;8:256-65.

22. Bigby C, Fyffe C. Tensions between institutional closure and deinstitutionalisation: what can be learned from Victoria's institutional redevelopment? Disabil Soc 2006;21:567-81.

23. Beadle-Brown J, Mansell J, Kozma A. Deinstitutionalization in intellectual disabilities. Curr Opin Psychiatry 2007;20:437-42.

24. Burrell B, Trip H. Reform and community care: has deinstitutionalisation delivered for people with intellectual disability? Nurs Inq 2011;18:174-83.

25. Wiesel I, Bigby C. Movement on shifting sands: deinstitutionalisation and people with intellectual disability in Australia, 1974-2014. Urban Policy and Research 2015;33:178-94.

26. Cochrane handbook. In: Higgins JPT, Green S, Cochrane handbook for systematic reviews of interventions version 5.1.0. edn, 2011.

27. Pope C, Ziebland S, Mays N. Analysing qualitative data. BMJ 2000;320:114-6.

28. Secondary. National disability authority of Ireland. $2018 \mathrm{http}: / / \mathrm{nda}$.ie/

29. Ager A, Myers F, Kerr P, et al. Moving home: social integration for adults with intellectual disabilities resettling into community provision. $J$ of Applied Research in Intellectual Disabilities 2001;14:392-400.

30. Barber JG, Cooper BK, Owen L. The short-term effects of relocation on the intellectually disabled. Res Soc Work Pract 1994;4:248-58.

31. Cooper B, Picton C. The long-term effects of relocation on people with an intellectual disability: quality of life, behavior, and environment. Res Soc Work Pract 2000;10:195-208.
32. Golding L, Emerson E, Thornton A. An evaluation of specialized community-based residential supports for people with challenging behaviour. J Intellect Disabil 2005;9:145-54.

33. Howard S, Spencer A. Effects of resettlement on people with learning disabilities. Br J Nurs 1997;6:436-41.

34. Young L. Community and cluster centre residential services for adults with intellectual disability: long-term results from an Australianmatched sample. J Intellect Disabil Res 2006;50:419-31.

35. Young L, Ashman AF. Deinstitutionalisation in Australia Part II: results from a long-term study. The British Journal of Development Disabilities 2004;50:29-45.

36. Young L, Ashman AF. Deinstitutionalization for older adults with severe mental retardation: results from Australia. Am J Ment Retard 2004;109:397-412.

37. Kilroy S, Egan J, Walsh M, et al. Staff perceptions of the quality of life of individuals with an intellectual disability who transition from a residential campus to community living in ireland: an exploratory study. Journal of Intellectual and Developmental Disability 2015;40:68-77.

38. Sheerin F, Griffiths C, de Vries J, et al. An evaluation of a community living initiative in Ireland. J Intellect Disabil 2015;19:266-81.

39. O'Brien P, Thesing A, Tuck B, et al. Perceptions of change, advantage and quality of life for people with intellectual disability who left a long stay institution to live in the community. $J$ Intellect Dev Disabil 2001;26:67-82.

40. Bigby C. Known well by no-one: trends in the informal social networks of middle-aged and older people with intellectual disability five years after moving to the community. J Intellect Dev Disabil 2008;33:148-57.

41. Di Terlizzi M. Life history: the impact of a changing service provision on an individual with learning disabilities. Disabil Soc 1994;9:501-17.

42. Ager A. The Life Experiences Checklist Part 1. Applications in staff training and programme planning. Journal of the British Institute of Mental Handicap 1993;21:7-9.

43. Schalock RL, Keith KD, Hoffman K. Quality of life questionnaire: standardization manual. Hastings, Nebraska: Mid-Nebraska Mental Retardation Services, 1990.

44. Working Group on Congregated Settings.. Time to move on from congregated settings: a strategy for community Inclusion. Dublin: Health Service Executive, 2011.

45. Kozma A, Mansell J, Beadle-Brown J. Outcomes in different residential settings for people with intellectual disability: a systematic review. Am J Intellect Dev Disabil 2009;114:193-222.

46. Felce D. Community living for adults with intellectual disabilities: unravelling the cost effectiveness discourse. J Policy Pract Intellect Disabil 2017;14:187-97.

47. Mansell J, Beadle-Brown J. Dispersed or clustered housing for adults with intellectual disability: a systematic review. J Intellect Dev Disabil 2009;34:313-23.

48. Chowdhury M, Benson BA. Deinstitutionalization and quality of life of individuals with intellectual disability: a review of the international literature. J Policy Pract Intellect Disabil 2011;8:256-65.

49. Martin L, Ashworth M, Ontario D. Canada: understanding who moved when. $J$ of Policy and Practice in Intellectual Disabilities 2010;7:167-76. 